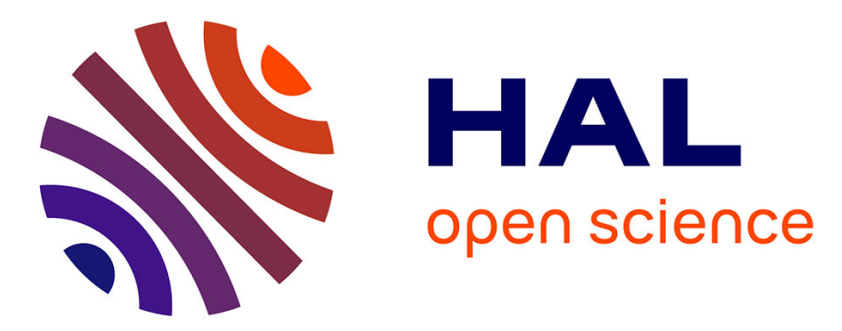

\title{
Un réferentiel typologique des habitats pour les régions Bretagne, Basse-Normandie et Pays-de-la-Loire
}

\author{
Loïc Delassus, Marion Hardegen
}

\section{To cite this version:}

Loïc Delassus, Marion Hardegen. Un réferentiel typologique des habitats pour les régions Bretagne, Basse-Normandie et Pays-de-la-Loire. Revue forestière française, 2010, 62 (3-4), pp.317-322. 10.4267/2042/38946 . hal-03449578

\section{HAL Id: hal-03449578 \\ https://hal.science/hal-03449578}

Submitted on 25 Nov 2021

HAL is a multi-disciplinary open access archive for the deposit and dissemination of scientific research documents, whether they are published or not. The documents may come from teaching and research institutions in France or abroad, or from public or private research centers.
L'archive ouverte pluridisciplinaire HAL, est destinée au dépôt et à la diffusion de documents scientifiques de niveau recherche, publiés ou non, émanant des établissements d'enseignement et de recherche français ou étrangers, des laboratoires publics ou privés. 


\title{
UN RÉFERENTIEL TYPOLOGIQUE DES HABITATS POUR LES RÉGIONS BRETAGNE, BASSE-NORMANDIE et PAYS-DE-LA-LOIRE
}

\author{
Loïc Delassus - MARION HARdegen
}

\section{HISTORIQUE DU PROJET}

En avril 1998, la DIREN Bretagne sollicitait le Conservatoire botanique national de Brest (CBNB) pour la réalisation d'inventaires et de cartographies d'habitats dans plusieurs sites Natura 2000 de Bretagne. Jugeant le cadre méthodologique existant au niveau national insuffisant, il avait été décidé d'engager une étude expérimentale sur l'inventaire et la cartographie des habitats. Cette étude devait permettre de répondre à un certain nombre de questions préliminaires d'ordre méthodologique et de tester différentes méthodes d'inventaire et de cartographie, afin d'identifier des protocoles opérationnels transposables à l'ensemble des sites Natura 2000 de Bretagne (cf. figure 1, p. 318).

Les réflexions sur la typologie des habitats à retenir pour la cartographie des habitats ont été au centre des préoccupations de cette étude expérimentale. En effet, toute représentation cartographique de la végétation suppose que, au préalable, les unités de végétation identifiées sur le terrain aient été classées selon une typologie adaptée aux réalités de terrain et à une transposition cartographique synthétique et pédagogique. Pour un programme homogène de cartographie de la végétation, il est ainsi indispensable de définir une typologie commune et claire qui permette de réduire les éventuelles différences d'interprétation lors de la phase d'inventaire et de pouvoir, in fine, comparer les différentes cartographies entre elles (dans l'espace et dans le temps).

Au cours de l'étude expérimentale, il est apparu préférable de privilégier une approche relativement fine du terrain, permettant d'obtenir des diagnostics de meilleure qualité et garantissant une plus grande possibilité d'évaluation a posteriori. Le choix s'est porté sur la typologie phytosociologique sigmatiste pour la description des unités de végétation, choix qui a été conforté à l'occasion d'un séminaire national consacré à la cartographie des habitats à Gap en 1999. Il tenait également compte des outils en cours de développement au niveau national, notamment les cahiers d'habitats nationaux, qui privilégiaient également l'approche phytosociologique pour la description des habitats d'intérêt communautaire.

Conscient que les typologies européennes CORINE Biotopes et EUR15 étaient très utilisées par les structures chargées de cartographier la végétation et qu'elles restaient une référence au niveau national et européen, il a paru important d'établir des liens entre la classification phytosociologique et ces différentes classifications européennes.

La première version du référentiel typologique, établie par le CBN de Brest en 2000, correspondait à une synthèse des groupements végétaux supposés présents en Bretagne d'après la biblio- 


\section{DU CONSERVATOIRE BOTANIQUE DE BREST DEPUIS 1999}

\begin{tabular}{|c|c|c|c|c|c|}
\hline \multicolumn{4}{|l|}{1999} & \multicolumn{2}{|c|}{2008} \\
\hline Texte & \multicolumn{3}{|c|}{ Base de données Access } & BD compatible Internet & Format \\
\hline \multirow{2}{*}{\multicolumn{2}{|c|}{$\begin{array}{l}\text { Phytosociologie } \\
\text { CORINE Biotopes } \\
\text { Habitats génériques N2000 }\end{array}$}} & \multicolumn{2}{|c|}{$\begin{array}{l}\text { + Habitats déclinés } \\
\text { N2000 }\end{array}$} & + EUNIS & Typologies \\
\hline & & $\begin{array}{l}\text { Références } \\
\text { bibliographiques }\end{array}$ & $\begin{array}{l}\text { Données } \\
\text { descriptives }\end{array}$ & $\begin{array}{l}\text { Espèces } \\
\text { caractéristiques }\end{array}$ & $\begin{array}{l}\text { Informations } \\
\text { complémentaires }\end{array}$ \\
\hline Bretagr & & \multicolumn{2}{|l|}{$\begin{array}{l}\text { Bretagne } \\
\text { Basse-Normandie } \\
\text { Pays de la Loire }\end{array}$} & & $\begin{array}{l}\text { Régions } \\
\text { concernées }\end{array}$ \\
\hline \multicolumn{2}{|c|}{$\begin{array}{l}\text { Envois ciblés chargés } \\
\text { d'inv. Natura } 2000\end{array}$} & \multicolumn{2}{|c|}{$\begin{array}{l}\text { Chargés d'inventaire N2000 des } \\
3 \text { Régions, autres partenaires (CG) }\end{array}$} & Mise en ligne & $\begin{array}{l}\text { Modes de } \\
\text { diffusion }\end{array}$ \\
\hline \multicolumn{2}{|c|}{ Outil de codification } & \multicolumn{3}{|c|}{ Outil de connaissance } & Type d'outil \\
\hline
\end{tabular}

graphie. Les travaux préliminaires au prodrome des végétations de France (colloque phytosociologique d'Orsay, 1996) menés en Bretagne par un groupe d'experts autour de Frédéric Bioret, Jan-Bernard Bouzillé, Bernard Clément, Jacques Haury et Jean Touffet (" prodrome des végétations armoricaines ») ont largement alimenté cette synthèse.

Pour chaque association végétale était ensuite proposé un (ou plusieurs) rattachement(s) aux codes européens CORINE Biotopes ainsi qu'aux habitats génériques de la directive habitats-faune-flore. Cette première version du référentiel typologique s'adressait aux chargés d'inventaire et de cartographie travaillant sur les sites Natura 2000, en Bretagne majoritairement des bureaux d'études.

Entre 2000 et 2004, l'outil "référentiel typologique" a progressivement évolué : son champ géographique s'est étendu pour couvrir tout le territoire d'agrément du Conservatoire botanique national de Brest, c'est-à-dire la Basse-Normandie, la Bretagne et les Pays-de-la-Loire (hors département de la Sarthe). D'autres typologies ont été ajoutées pour tenir compte des évolutions aux niveaux national et européen : codes EUNIS et habitats déclinés des cahiers d'habitats français. La parution du prodrome des végétations de France (Bardat et al., 2004) a rendu nécessaire quelques modifications concernant le synsystème pour assurer la cohérence entre le référentiel typologique et les choix de synsystème faits dans le prodrome.

Une autre évolution technique a été la construction d'une base de données Access ${ }^{\circledR}$ centralisant toutes les informations du référentiel ; la consultation du référentiel a ainsi été largement facilitée.

Par ailleurs, pour tenir compte des souhaits des utilisateurs du référentiel, de plus en plus nombreux et de profils diversifiés (chargés d'inventaire et de cartographie, opérateurs locaux Natura 2000, gestionnaires d'espaces naturels...), il a été décidé de faire évoluer le référentiel pour le faire passer d'un outil de "codification" à un outil de connaissance des habitats présents sur le territoire d'agrément du CBN de Brest. En effet, le simple fait de "lister" les groupements végétaux n'apportait aucune information sur les caractéristiques diagnostiques des groupements et réduisait le champ des utilisateurs à un public d'initiés, ayant accès à la bibliographie spécialisée. 
C'est ainsi que peu à peu des informations descriptives concernant les groupements végétaux cités ont été intégrées : espèces caractéristiques, petit descriptif de la physionomie et de l'écologie et liste des références bibliographiques concernant la communauté végétale disponibles au CBN de Brest.

\section{LE RÉFÉRENTIEL TYPOLOGIQUE AUJOURD’HUI}

\section{Nombre de syntaxons recensés}

Aujourd'hui, le référentiel typologique du CBN de Brest peut être considéré comme le synopsis des végétations naturelles et semi-naturelles de Basse-Normandie, Bretagne et Pays-de-la-Loire. Actuellement, 755 syntaxons du niveau de l'association sont intégrés au référentiel typologique. Ils sont organisés dans le synsystème suivant le Prodrome des végétations de France selon 58 classes, 93 ordres et 191 alliances.

Pour chacun des syntaxons, il est proposé une description, les correspondances avec les différentes typologies européennes ainsi qu'une liste de références bibliographiques à laquelle il est possible de se reporter. Ces données sont consultables sur le site internet du CBN de Brest (figure 2, p. 320) : http://www.cbnbrest.fr/site/Refer_typo/habito.php.

\section{Validation synnomenclaturale et synsystématique}

Si les niveaux supérieurs à l'association ont fait l'objet d'une validation synnomenclaturale et synsystématique selon le code de nomenclature lors de la rédaction du Prodrome des végétations de France, il n'en va pas de même pour les associations citées dans le référentiel typologique. L'outil reflète tout de même l'état des connaissances actuelles en matière de communautés végétales et met en évidence les lacunes. De plus, bien que le référentiel soit apprécié, il ne remplace pas encore la bibliographie. En effet, toutes les informations entrées (descriptifs, espèces indicatrices, etc.) sont peu homogènes et souvent incomplètes. Elles demandent donc à être consolidées et harmonisées.

\section{Un outil au service des chargés d'inventaire et de cartographie}

Le référentiel typologique du CBN de Brest est un outil très apprécié par les personnes en charge d'inventaires et de cartographies de la végétation, comme le montrent les nombreuses consultations de la page dédiée au référentiel sur le site internet du CBN de Brest. Elle est en effet une des pages les plus consultées du site. Les visiteurs y recherchent un outil leur permettant une première approche pour l'identification des communautés végétales ainsi que pour la mise en évidence de la présence de certains types d'habitats (au sens européen) sur leurs sites. Ceci montre bien l'intérêt que portent les acteurs de terrain à un outil de centralisation et de diffusion des données sur les communautés végétales du territoire d'agrément.

La conception du référentiel typologique des habitats naturels et semi-naturels de BasseNormandie, Bretagne et Pays-de-la-Loire s'est ainsi inscrite depuis le début dans la nécessité de proposer aux personnes chargées de réaliser des cartographies d'habitats un outil opérationnel ayant un lien fort avec la réalité de terrain. Cependant, l'entrée "nomenclature phytosociologique" peut parfois faire peur aux chargés d'études. Il apparaît ainsi qu'un des défis pour la phytosociologie va se situer dans la capacité à communiquer autour de cette discipline. C'est dans ce cadre qu'une liste de référence des habitats en français est en cours de réalisation par le CBN. Cette liste de référence se base sur le synsystème et propose une typologie emboîtée articulée autour des grands types de milieux. L'objectif est également de valoriser les données 
Loïc Delassus - Marion Hardegen

de la phytosociologie auprès des instances en charge de l'environnement de l'État ou des collectivités territoriales. Pour être pertinente, la liste doit être cohérente par rapport aux différentes typologies européennes.

FIGURE 2 CONSULTATION DU RÉFÉRENTIEL TYPOLOGIQUE VIA INTERNET

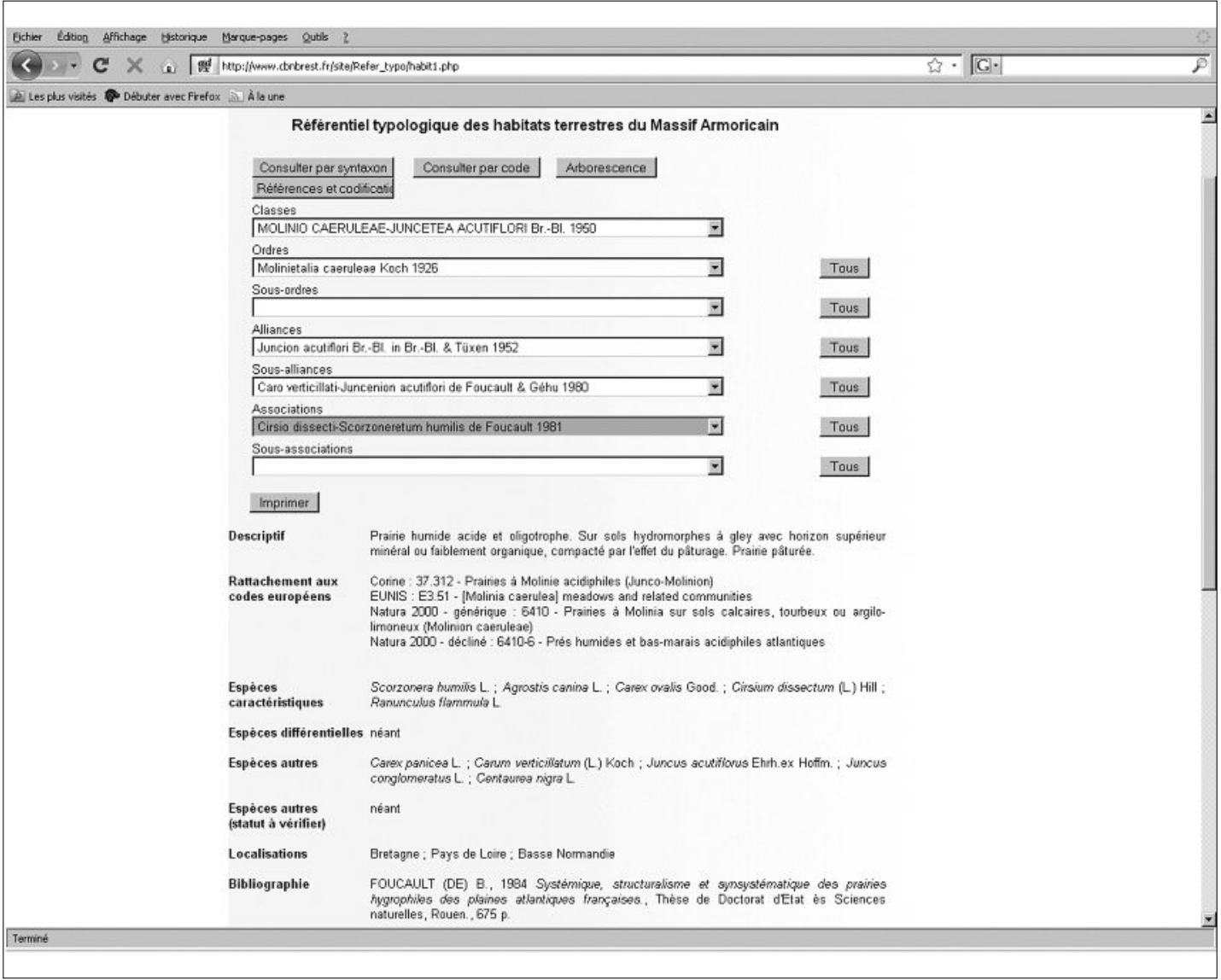

\section{LE RÉFÉRENTIEL TYPOLOGIQUE AU CENTRE DES PROJETS FUTURS SUR LES HABITATS}

S'il est vrai qu'un gros travail de recherche et de centralisation des données sur les communautés végétales de Basse-Normandie, Bretagne et des Pays-de-la-Loire a été réalisé dans le cadre du référentiel typologique du CBN de Brest, force est de constater qu'il existe aujourd'hui de grosses lacunes dans la connaissance des végétations et de leur répartition. Or, l'État et les collectivités territoriales prennent de plus en plus en compte la dimension habitats dans leur stratégie de préservation de la biodiversité. Il apparaît donc qu'il est souvent difficile de répondre aujourd'hui objectivement aux questions de vulnérabilité, d'état de conservation ou de distribution des habitats. 
Ainsi, dans la mesure où, dans la majorité des cas, on se fonde sur la description des communautés végétales pour définir les habitats, le référentiel typologique des habitats naturels et semi-naturels de Basse-Normandie, Bretagne et des Pays-de-la-Loire se place comme une pièce centrale dans un programme d'amélioration de la connaissance des habitats. Pour remplir ce rôle, l'outil doit évoluer et être consolidé, les données doivent être confortées et harmonisées et l'accessibilité optimisée.

Loïc DELASSUS - Marion HARDEGEN

CONSERVATOIRE BOTANIQUE NATIONAL DE BREST

52 allée du Bot

F-29200 BREST

(l.delassus@cbnbrest.com)

(m.hardegen@cbnbrest.com)

\section{BIBLIOGRAPHIE}

BARDAT (J.), BIORET (F.), BOTINEAU (M.), BOULLET (V.), DELPECH (R.), GÉHU (J.-M.), HAURY (.), LACOSTE (A.), RAMEAU (..-C.), ROYER (..-M.), ROUX (G.), TOUFFET (..). - Prodrome des végétations de France. - Paris : Muséum national d'histoire naturelle, 2004. - 171 p. (Patrimoines naturels, 61).

BENSETTITI (F.), (coord.). - Connaissance et gestion des habitats et des espèces d'intérêt communautaire. Tome 1 : Habitats forestiers, volume 1. - Paris : La Documentation française, 2001. - $339 \mathrm{p}$.

BENSETTITI (F.), (coord.). - Connaissance et gestion des habitats et des espèces d'intérêt communautaire. Tome 1 : Habitats forestiers, volume 2. - Paris : La Documentation française, 2001. - $423 \mathrm{p}$.

BENSETTITI (F.), (coord.). - Connaissance et gestion des habitats et des espèces d'intérêt communautaire. Tome 2 : Habitats côtiers. Rapport pour le ministère de l'Environnement et du Développement durable. - Paris : La Documentation française, 2004. - 399 p.

BENSETTITI (F.), (coord.). - Connaissance et gestion des habitats et des espèces d'intérêt communautaire. Tome 3 : Habitats humides. - Paris : La Documentation française, 2002. - 457 p.

BENSETTITI (F.), (coord.). - Connaissance et gestion des habitats et des espèces d'intérêt communautaire. Tome 4 : Habitats agropastoraux, volume 1. - Paris : La Documentation française, 2005. - $445 \mathrm{p}$.

BENSETTITI (F.), (coord.). - Connaissance et gestion des habitats et des espèces d'intérêt communautaire. Tome 4 : Habitats agropastoraux, volume 2. - Paris : La Documentation française, 2005. - $487 \mathrm{p}$.

BENSETTITI (F.), (coord.). - Connaissance et gestion des habitats et des espèces d'intérêt communautaire. Tome 5 : Habitats rocheux. Rapport pour le ministère de l'Environnement et du Développement durable. - Paris : La Documentation française, 2004. - $381 \mathrm{p}$.

BISSARDON (M.), GUIBAL (L.), sous la direction de RAMEAU (J.-C.). - Nomenclature Corine Biotopes, Version originale, types d'habitats français. - Nancy : ENGREF, 1997. - 219 p.

COMMISSION EUROPÉENNE, DIRECTION GÉNÉRALE DE L'ENVIRONNEMENT. - Interpretation Manual of European Union Habitats. EUR 25, avril 2003. - 127 p.

MOSS (D.), DAVIES (C.E.). - EUNIS Habitat Classification. - Huntingdon (Camb., UK) : European Environnemental Agency - European Topic Center of Nature Conservation and Biodiversity - Center of Ecology and Hydrology, 2002. 
Loïc Delassus - Marion Hardegen

UN RÉFERENTIEL TYPOLOGIOUE DES HABITATS POUR LES RÉGIONS BRETAGNE, BASSE-NORMANDIE ET PAYS-DE-LA-LOIRE [Résumé]

Tout travail d'inventaire nécessite de connaître son objet d'étude. S'il existe des flores pour la détermination des espèces végétales, il n'en est pas de même pour les groupements végétaux. L'identification de ces derniers nécessite en général la consultation d'un grand nombre de publications scientifiques, qui ne sont pas toujours faciles d'accès. Les Cahiers d'habitats comblent en partie ce déficit pour les habitats d'intérêt communautaire (parution échelonnée entre 2002 et 2005), mais pour les autres habitats il existe très peu, voire pas, de référence synthétique fournissant une aide à la détermination. Pour commencer à pallier ces lacunes, le Conservatoire botanique national de Brest a développé depuis 2000 un outil visant à référencer l'ensemble des communautés végétales présentes sur sa zone d'intervention : la Bretagne, la BasseNormandie et les Pays-de-la-Loire.

Ainsi, en partant des typologies d'habitats existantes - nomenclature phytosociologique, CORINE Biotopes, EUNIS, codes Natura 2000 et habitats élémentaires des Cahiers d'habitats - le CBN de Brest a développé un référentiel typologique qui établit les correspondances entre la nomenclature phytosociologique et les différentes codifications européennes et nationales. En même temps, ce référentiel tente de dresser la liste des associations végétales présentes sur son territoire d'agrément et contribue ainsi à une première synthèse de la connaissance sur les habitats.

\section{A TYPOLOGICAL CLASSIFICATION FOA HABITATS IN THE BRETAGNE, LOWER NOAMANDY AND PAYS-DE-LA-LOIRE REGIONS [Abstract]}

Any inventory exercise requires proper knowledge about its object. Floras exist for the determination of plant species, but no similar tool exists for plant communities. As a rule, innumerable scientific publications must be consulted that are not always readily accessible. The Cahiers d'Habitats partially overcome this shortcoming as regards European-interest habitats (published by instalments between 2002 and 2005), but for other habitats there are very few and sometimes no synoptic classifications that can be used to assist in identification. To overcome these shortcoming, the National Botanical Conservancy of Brest began to develop a tool in 2000 for the purpose of classifying all the plant communities present in the area under its scope: Brittany, Lower Normandy and the Pays-de-la-Loire.

Starting out from existing habitat typologies - phytosociological nomenclature, CORINE Biotopes, EUNIS, Natura 2000 codes and the elementary habitats in the Cahiers d'habitats - the Brest Conservancy has developed a standard typology that crossmatches the phytosociological nomenclature and the various European and national coding systems. The aim of this typology to draw up a list of the vegetal associations found within Conservancy's geographical area thereby contributing to a first synopsis of current knowledge about these habitats. 